\title{
EQUIVARIANT $K$-THEORY AND REPRESENTATIONS OF HECKE ALGEBRAS
}

\author{
GEORGE LUSZTIG ${ }^{1}$
}

\begin{abstract}
We construct some representations of the Hecke algebra of an affine Weyl group using equivariant $K$-theory and state a conjecture on some $q$-analogs of the Springer representations.
\end{abstract}

1. This paper contains a new construction of the principal series representations of the Hecke algebra of an affine Weyl group, in terms of the equivariant $K$-theory of a flag manifold. The formulas defining the simplest operators in these representations are $q$-analogs of operators considered earlier by Demazure [1]. The results in this paper were found during a visit to the Tata Institute of Fundamental Research, Bombay, in December 1983; I am grateful to D. N. Verma for some stimulating discussions.

2. We recall (cf. Segal [4]) that if $X$ is a compact topological space with a continuous action of a compact topological group $M^{\prime}$, then the equivariant $K$-theory $K_{M^{\prime}}(X)$ is defined as the Grothendieck group of the category whose objects are the $M^{\prime}$-equivariant complex vector bundles on $X$ and the morphisms are $M^{\prime}$-equivariant maps with locally constant rank. Then $K_{M^{\prime}}(X)$ is naturally an $R_{M^{\prime}}$-module where $R_{M^{\prime}}=K_{M^{\prime}}$ (point) is the representation ring of $M^{\prime}$, i.e. the Grothendieck group of the category of finite dimensional continuous complex representations of $M^{\prime}$.

3. We shall need a variant of this definition, in which $M^{\prime}$ is replaced by a complex Lie group $M$ underlying a (not necessarily connected) reductive complex algebraic group. We assume that $M$ acts continuously on the compact topological space $X$, and we wish to define $K_{M}(X)$.

According to Mostow (see [2, Chapter XV]), $M$ has maximal compact subgroups, any two such are conjugate and any connected component of the normalizer in $M$ of a maximal compact subgroup $M^{\prime}$, meets $M^{\prime}$. We shall construct, for any two maximal compact subgroups $M^{\prime}$ and $M^{\prime \prime}$ of $M$, a canonical isomorphism $\phi_{M^{\prime}, M^{\prime \prime}}: K_{M^{\prime}}(X) \stackrel{\sim}{\rightarrow} K_{M^{\prime \prime}}(X)$ as follows. Choose $g \in M$ such that $g M^{\prime \prime} g^{-1}=M^{\prime}$. If $E$ is an $M^{\prime}$-equivariant vector bundle on $X$, we define a new vector bundle $g^{*} E$ on $X$ as the inverse image of $E$ under the map $X \rightarrow X, x \mapsto g x$. It is clear that $g^{*} E$ is naturally an $M^{\prime \prime}$-equivariant vector bundle on $X$ and $\phi_{M^{\prime}, M^{\prime \prime}}$ is defined by $E \mapsto g^{*} E$. To show that $\phi_{M^{\prime}, M^{\prime \prime}}$ is independent of the choice of $g$, we may assume that $M^{\prime}=M^{\prime \prime}$ and that $g \in M$ normalizes $M^{\prime}$; we must show that $g^{*} E \approx E$ as $M^{\prime}$-equivariant vector bundles. Since the isomorphism class of $g^{*} E$ does not change when $g$ runs through a fixed connected component of the normalizer of $M^{\prime}$, and since $M^{\prime}$ meets each such component, we can further assume that $g \in M^{\prime}$. In this

Received by the editors August 12, 1984.

1980 Mathematics Subject Classification. Primary 22E50; Secondary 18F25, 16A99.

${ }^{1}$ Supported in part by the National Science Foundation. 
case, the $M^{\prime}$-equivariant structure of $E$ defines an isomorphism $E_{x} \stackrel{g}{\rightarrow} E_{g x}=\left(g^{*} E\right)_{x}$ for all $x \in X$ and hence an isomorphism $g^{*} E \approx E$ as desired.

The isomorphisms $\phi_{M^{\prime}, M^{\prime \prime}}$ have an obvious transitivity property. We may therefore define $K_{M}(X)$ to be $\lim _{M^{\prime}}(X)$ (limit over all maximal compact subgroups $M^{\prime}$ of $M$, with respect to the isomorphisms $\left.\phi_{M^{\prime}, M^{\prime \prime}}\right)$. Then we have natural isomorphisms $K(X) \stackrel{\sim}{\rightarrow} K_{M^{\prime}}(X)$ for any maximal compact subgroup $M^{\prime} \subset M$. It also follows that $K_{M}(X)$ is naturally an $R_{M}$-module where $R_{M}=K_{M}$ (point) is the representation ring of $M$, i.e. the Grothedieck group of the category of finite dimensional complex algebraic representations of $M$. (Note that $R_{M} \stackrel{\sim}{\rightarrow} R_{M^{\prime}}$ for any maximal compact subgroup $M^{\prime} \subset M$.)

4. We now consider a simple, simply connected complex algebraic group $G$ and $X=G / B$, where $B$ is a Borel subgroup of $G$. Then $M=G \times \mathbf{C}^{*}$ acts on $X$ as follows: $G$ acts by left translation and $C^{*}$ acts trivially. We have $K_{M}(X)=$ $K_{G}(X) \otimes R_{\mathbf{C}^{*}}=K_{G}(X) \otimes Z\left[q, q^{-1}\right]$, where $q$ is the generator of $R_{\mathbf{C}^{*}}$ corresponding to the identity representation $\mathbf{C}^{*} \stackrel{\sim}{\rightarrow} \mathbf{C}^{*}$.

Let $T$ be a maximal torus in $B, W$ the Weyl group of $G$ with respect to $T, S$ the set of simple reflections in $W$ (with respect to $B$ ), $P$ the lattice of weights $T \rightarrow \mathbf{C}^{*}$, $R \subset P$ the set of roots and $R^{+}$the set of positive roots (with respect to $B$ ).

For each $s \in S$, let $P_{s}$ be the parabolic subgroup $B \cup B s B$ and let $\pi_{s}: X \rightarrow G / P_{s}$ be the natural map. There is a unique endomorphism

$$
T_{s}: K_{M}(X) \rightarrow K_{M}(X)
$$

with the following property: if $E$ is an $M$-equivariant algebraic vector bundle on $X$, then

$$
E+T_{s} E=\pi_{s}^{*}\left(\pi_{s}\right)_{*}(E)-\pi_{s}^{*}\left(\pi_{s}\right)_{*}\left(E \otimes \Omega_{s}^{1}\right),
$$

where $\Omega_{s}^{1}$ is the line bundle on $X$ of holomorphic differential 1-forms along the fibres of $\pi_{s}$, regarded as an $M$-equivariant bundle with the obvious action of $G$ and with the action of $\mathbf{C}^{*}$ given by scalar multiplication on each fibre of $\Omega_{s}^{1}$. Here $\left(\pi_{s}\right)_{*}(E)$ is the alternating sum of the higher direct images of $E$ under $\pi_{s}$ in the category of coherent sheaves; these higher direct images are again $M$-equivariant algebraic vector bundles on $G / P_{s}$, hence their alternating sum defines an element in $K_{M}\left(G / P_{s}\right)$.

For any weight $p \in P$, we define an endomorphism

$$
\theta_{p}: K_{M}(X) \rightarrow K_{M}(X)
$$

by

$$
\theta_{p} E=E \otimes L_{p}^{*}
$$

where $L_{p}$ is the line bundle on $X=G / B$ associated to the homomorphism $B \rightarrow \mathbf{C}^{*}$ obtained by composing the projection $B \rightarrow T$ with $p: T \rightarrow \mathbf{C}^{*}$, and $L_{p}^{*}$ is the dual line bundle; we regard $L_{p}^{*}$ as an $M$-equivariant bundle with the obvious action of $G$ and with trivial action of $\mathbf{C}^{*}$.

5. The group structure on the lattice of weights $P$ will be written multiplicatively. The Weyl group $W$ acts naturally on $P(w: p \rightarrow w(p))$ and we form the semidirect product $\tilde{W}=W \cdot P$ with $P$ normal and $w \cdot p=w(p) \cdot w(w \in W, p \in P)$. 
Then $\tilde{W}$ contains the affine Weyl group as a subgroup of finite index. According to Bernstein (see $[3,4.4]$ ) one can describe the Hecke algebra $\tilde{H}$ corresponding to $\tilde{W}$ as follows. It is an algebra over $\mathbf{Z}\left[q, q^{-1}\right]$ with generators $T_{s}(s \in S)$ and $\theta_{p}(p \in P)$ subject to the following relations:

$$
\begin{gathered}
\left(T_{s}+1\right)\left(T_{s}-q\right)=0 \quad(s \in S), \\
T_{s} T_{t} T_{s} \cdots=T_{t} T_{s} T_{t} \cdots
\end{gathered}
$$

$\left(s \neq t \in S\right.$; both sides have $m_{s, t}$ factors where $m_{s, t}=$ order of $s t$ in $W$ ),

$$
\begin{gathered}
\theta_{p} \theta_{p^{\prime}}=\theta_{p p^{\prime}} \quad\left(p, p^{\prime} \in P\right), \\
T_{s} \theta_{p}=\theta_{p} T_{s} \quad(s \in S, p \in P, s p=p s), \\
T_{s} \theta_{s(p)} T_{s}=q \theta_{p} \quad\left(s \in S, p \in P, s p s^{-1} p^{-1}=\alpha_{s}^{-1}\right) .
\end{gathered}
$$

Here $\alpha_{s} \in R^{+} \subset P$ is the simple root corresponding to $s$. We can now state our main result.

THEOREM. The endomorphisms $T_{s}, \theta_{p}$ of $K_{M}(X)$ defined in $\S 4$ give rise to a left $\tilde{H}$-module structure on $K_{M}(X)$. (The action of $\mathbf{Z}\left[q, q^{-1}\right] \subset \tilde{H}$ is defined to be the same as the restriction to $R_{C^{*}}$ of the earlier action of $R_{M}$.) This $\tilde{H}$-module structure commutes with the $R_{M}$-module structure on $K_{M}(X)$.

The proof will be given in $\S 8.1$.

6. We now fix a unipotent element $u \in G$. Let $\phi: \mathrm{SL}_{2}(\mathbf{C}) \rightarrow G$ be an algebraic homomorphism such that $u=\phi\left(\begin{array}{ll}1 & 1 \\ 0 & 1\end{array}\right)$ and let $D$ be the group of diagonal matrices in $\mathrm{SL}_{2}(\mathbf{C})$. We define

$$
M_{u}=\left\{(g, \lambda) \in G \times \mathbf{C}^{*} \mid g^{-1} u g=u^{\lambda}, g \in Z_{G}(\phi(D))\right\} .
$$

(Note that any complex power of a unipotent element is well defined.) Then $M_{u}$ is a reductive algebraic subgroup of $G \times \mathbf{C}^{*}$; if $u=e$ then $M_{u}=M$ of $\S 4$. Let $X_{u}=\left\{g_{1} B \in X \mid g_{1}^{-1} u g_{1} \in B\right\} ;$ it is a closed subvariety of $X=G / B$ with an action of $M_{u}$ given by $(g, \lambda): g_{1} B \rightarrow g g_{1} B$. We now fix a connected component $c$ of $M_{u}$ and let $M_{u, c}$ be the inverse image under $M_{u} \rightarrow M_{u} / M_{u}^{0}$ of the cyclic group in $M_{u} / M_{u}^{0}$ generated by the image of $c$. The $R_{M_{u, c}}$-module $K_{M_{u, c}}\left(X_{u}\right)$ is defined as in $\S 2$. Restriction of vector bundles gives rise to a homomorphism of $R_{M_{u, c}}$-modules

$$
R_{M_{u, c}} \otimes_{R_{M}} K_{M}(X) \rightarrow K_{M_{u, c}}\left(X_{u}\right) .
$$

(We regard $R_{M_{u, c}}$ as an $R_{M}$-module, via the homomorphism $R_{M} \rightarrow R_{M_{u, c}}$ induced by the inclusion $M_{u, c} \subset M$.) Now let $\bar{R}_{M_{u, c}}$ be the ring $R_{M_{u, c}} / J$, where $J$ is the ideal of $R_{M_{u, c}}$ consisting of all $E \in R_{M_{u, c}}$ whose character is identically zero on $c$. Then from (6.1) we get a homomorphism of $\bar{R}_{M_{u, c}}$-modules

$$
\bar{R}_{M_{u, c}} \otimes_{R_{M}} K_{M}(X) \rightarrow \bar{R}_{M_{u, c}} \otimes_{R_{M_{u, c}}} K_{M_{u, c}}\left(X_{u}\right)
$$

We have a natural surjective homomorphism $M_{u, c} \rightarrow \mathbf{C}^{*},(g, \lambda) \rightarrow \lambda$; it induces a ring homomorphism $R_{\mathbf{C}^{*}} \rightarrow R_{M_{u, c}}$ and we shall denote the image of $q \in R_{\mathbf{C}^{*}}$ in $\bar{R}_{M_{u, c}}$ again by $q$. We can now state the following conjecture. 
CONJECTURE. There exists a natural left $\tilde{H}$-module structure on

$$
\bar{R}_{M_{u, c}} \otimes_{R_{M_{u, c}}} K_{M_{u, c}}\left(X_{u}\right)
$$

with the following properties:

(a) It is compatible under (6.2) with the $\tilde{H}$-module structure on

$$
\bar{R}_{M_{u, c}} \otimes_{R_{M}} K_{M}(X)
$$

deduced by extension of scalars from the $\tilde{H}$-module structure on $K_{M}(X)$ described in the Theorem.

(b) The action of $\theta_{p}(p \in P)$ on

$$
\bar{R}_{M_{u, c}} \otimes_{R_{M_{u, c}}} K_{M_{u, c}}\left(X_{u}\right)
$$

is by tensor product with the restriction of $L_{p}^{*}$ to $X_{u}$ (see (4.4)).

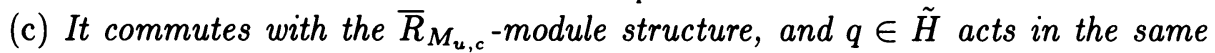
way as $q \in \bar{R}_{M_{u, c}}$.

7. Assuming the conjecture, let us consider a semisimple element $s \in G$ such that $\left(s, q_{0}\right) \in M_{u}$ for some $q_{0} \in \mathbf{C}^{*}$. Let $c$ be the component of $M_{u}$ containing $s$. Let

$$
\tilde{H}\left(q_{0}\right)=\mathbf{C} \otimes_{\mathbf{z}\left[q, q^{-1}\right]} \tilde{H},
$$

where $\mathbf{C}$ is regarded as a $\mathbf{Z}\left[q, q^{-1}\right]$-module with $q$ acting as multiplication by $q_{0}$. Let $h_{s}: R_{M_{u, c}} \rightarrow \mathbf{C}$ be the ring homomorphism defined by $E \rightarrow \operatorname{Tr}(s, E)(E \in$ $R_{M_{u, c}}$ ). This homomorphism factors through $\bar{R}_{M_{u, c}}$, since $s \in c$. Consider the tensor product

$$
F_{u, s}=\mathbf{C} \otimes_{R_{M_{u, c}}} K_{M_{u, c}}\left(X_{u}\right),
$$

where $\mathbf{C}$ is regarded as a $R_{M_{u, c}}$-module via $h_{s}$. Note that $F_{u, s}$ is a finite dimensional C-vector space. (Indeed by a theorem of Segal [4], $K_{M_{u, c}}\left(X_{u}\right)$ is a finitely generated $R_{M_{u, c}}$-module.) The conjecture implies that there is a natural left $\tilde{H}\left(q_{0}\right)$-module structure on $F_{u, s}$. It is a $q$-analog of the $W$-representations of Springer, which were extended to $\tilde{W}$ by S. Kato (Nederl. Akad. Wetensch. Proc. Ser. A 86 (1983), 193-201).

8. Proof of the theorem. It is well known that the elements $L_{p} \in K_{G}(X)$ $(p \in P)$ form a $\mathbf{Z}$-basis of $K_{G}(X)$. Thus $K_{G}(X)$ may be identified with the group ring $\mathbf{Z}[P]$. It is also known that under this identification the canonical ring homomorphism $R_{G} \rightarrow K_{G}(X)$ becomes the inclusion of the $W$-invariants $\mathbf{Z}[P]^{W}$ into $\mathbf{Z}[P]$. It follows that we may identify $K_{M}(X)$ with $R_{0}=$ group ring of $P$ over $\mathbf{Z}\left[q, q^{-1}\right]$ and the canonical ring homomorphism $R_{M} \rightarrow K_{M}(X)$ with the inclusion $R_{0}^{W} \rightarrow R_{0}$. We shall denote by $R$ the quotient field of $R_{0}$. With these identifications, the map $T_{s}: K_{M}(X) \rightarrow K_{M}(X)$ becomes the $\mathbf{Z}\left[q, q^{-1}\right]$-linear map $T_{s}: R_{0} \rightarrow R_{0}$ given by

$$
T_{s}(\lambda)=\frac{\lambda-s(\lambda)}{\alpha_{s}-1}-q \frac{\lambda-s(\lambda) \alpha_{s}}{\alpha_{s}-1} \quad(\lambda \in P) .
$$

Note that this is a priori an element of $R$. But it is easily seen that it is actually in $R_{0}$. (If we specialize $q$ to 1 this is just the action $\lambda \rightarrow s(\lambda)$ of $W$ on $P$. If we 
specialize $q$ to 0 we obtain essentially Demazure's operator [1]. Thus our operator (8.1) is the simplest possible interpolation between these two special cases.)

The map $\theta_{p}: K_{M}(X) \rightarrow K_{M}(X)$ becomes with the previous identifications the $Z\left[q, q^{-1}\right]$-linear map $\theta_{p}: R_{0} \rightarrow R_{0}$ defined by

$$
\theta_{p}(\lambda)=\lambda p^{-1} \text {. }
$$

We must show that the endomorphisms $(8.1),(8.2)$ of $R_{0}$ verify the identities (5.1)(5.5).

The identities (5.1), (5.3) and (5.4) are immediate. Now let $s \neq t$ be two simple reflections in $S$ and let $\langle s, t\rangle$ be the subgroup of $W$ they generate. Let $\Phi_{s, t}$ be the set of roots which are $\mathbf{Z}$-combinations of the simple roots $\alpha_{s}, \alpha_{t}$ and let $\Phi_{s, t}^{+} \cup \Phi_{s, t}^{-}$ be its partition into positive and negative roots. Let $\rho_{s, t}$ be the element of $P$ such that

$$
\rho_{s, t}^{2}=\prod_{\alpha \in \Phi_{s, t}^{+}} \alpha
$$

Let

$$
\Psi^{+}=\prod_{\alpha \in \Phi_{s, t}^{+}}(1-q \alpha), \quad \Psi^{-}=\prod_{\alpha \in \Phi_{s, t}^{-}}(1-q \alpha) .
$$

For any element $\xi \in R_{0}$ we define $\operatorname{Alt}_{s, t}(\xi)=\sum_{w \in\langle s, t\rangle}(-1)^{l(w)} w(\xi)$, where $l$ is the length function on $W$.

We denote the product $T_{s} T_{t} T_{s} \cdots$ ( $i$ factors) by $T_{s, i}$ and similarly we denote the product $T_{t} T_{s} T_{t} \cdots$ ( $i$ factors) by $T_{t, i}$. With these notations we state the following identity which is verified by direct computation. (Here $m=$ order of $s t$ in $W$.)

$$
\begin{aligned}
(1+ & \left.\sum_{1 \leq i \leq m} T_{s, i}+\sum_{1 \leq i \leq m-1} T_{t, i}\right)(\lambda) \\
& =\operatorname{Alt}_{s, t}\left(\lambda \rho_{s, t} \Psi^{-}\right) \cdot\left(\operatorname{Alt}_{s, t}\left(\rho_{s, t}\right)\right)^{-1} \quad(\lambda \in P) .
\end{aligned}
$$

The right-hand side of this identity is symmetric in $s, t$. Hence so is the left-hand side. It follows that $T_{s, m}=T_{t, m}$ and (5.2) follows. (Alternatively, one can use the following identity:

$$
\begin{gathered}
\left((-q)^{m}+\sum_{1 \leq i \leq m}(-q)^{m-i} T_{s, i}+\sum_{1 \leq i \leq m-1}(-q)^{m-i} T_{t, i}\right)(\lambda) \\
\left.=\rho_{s, t}^{-1} \Psi^{+} \operatorname{Alt}_{s, t}(\lambda) \cdot\left(\operatorname{Alt}_{s, t}\left(\rho_{s, t}\right)\right)^{-1} \quad(\lambda \in P) .\right)
\end{gathered}
$$

With the assumption of (5.5) we have for $\lambda \in P$ :

$$
\begin{aligned}
T_{s} \theta_{s(p)} T_{s}(\lambda) & =T_{s} \frac{\lambda-s(\lambda)-q\left(\lambda-s(\lambda) \alpha_{s}\right)}{\left(\alpha_{s}-1\right) s(p)} \\
& =\frac{1}{\alpha_{s}-1}\left(\frac{\lambda-s(\lambda)-q\left(\lambda-s(\lambda) \alpha_{s}\right)}{\left(\alpha_{s}-1\right) p \alpha_{s}^{-1}}(1-q)\right. \\
& \left.\quad-\frac{s(\lambda)-\lambda-q\left(s(\lambda)-\lambda \alpha_{s}^{-1}\right)}{\left(\alpha_{s}^{-1}-1\right) p}\left(1-q \alpha_{s}\right)\right) \\
& =q \lambda p^{-1}=q \theta_{p}(\lambda) .
\end{aligned}
$$


We now show that the operators $T_{s}, \theta_{p}: R_{0} \rightarrow R_{0}$ are $R_{0}^{W}$-linear. This is trivial for $\theta_{p}$. For $T_{s}$, we have the following multiplicative property (whose proof is trivial):

$$
T_{s}(f \cdot g)=\left(T_{s} f\right) \cdot g+s(f) \cdot T_{s}(g)-q s(f) \cdot g
$$

for any $f, g \in R_{0}$. If $f=s(f)$, then clearly $T_{s} f=q f$ so that $T_{s}(f \cdot g)=f \cdot T_{s}(g)$. In particular, if $f \in R_{0}^{W}$, then $T_{s}(f \cdot g)=f \cdot T_{s}(g)$ for all $g \in R_{0}$ and all $s \in S$. This shows that $T_{s}$ is $R_{0}^{W}$-linear.

9. Remarks. (a) By a theorem of Pittie, $R_{0}$ is a free $R_{0}^{W}$-module of rank $|W|$.

(b) The formula (8.3) has the following interpretation. Let $P_{s, t}$ be the parabolic subgroup $\bigcup_{w \in\langle s, t\rangle} B w B$ and let $\pi_{s, t}$ be the natural map $G / B \rightarrow G / P_{s, t}$. Let us define $T_{w}$ for $w \in W$ as $T_{s_{1}} T_{s_{2}} \cdots T_{s_{r}}$, where $s_{1} s_{2} \cdots s_{r}$ is a reduced expression for $W$. Then for any $M$-equivariant algebraic vector bundle $E$ on $X=G / B$, we have

$$
\sum_{w \in\langle s, t\rangle} T_{w} E=\sum_{i}(-1)^{i} \pi_{s, t}^{*}\left(\pi_{s, t}\right)_{*}\left(E \otimes \Omega_{s, t}^{i}\right),
$$

where $\Omega_{s, t}^{i}$ is the vector bundle on $X$ of holomorphic differential $i$-forms along the fibres of $\pi_{s, t}$ regarded as an $M$-equivariant bundle with the obvious action of $G$ and with the action of $\mathbf{C}^{*}$ given by scalar multiplication by $z^{i}$ on each fibre. (Compare (4.2).)

\section{REFERENCES}

1. M. Demazure, Une nouvelle formule des caractères, Bull. Soc. Math. France (2) 98 (1974), 163-172.

2. G. Hochschild, The structure of Lie groups, Holden-Day, San Francisco, Calif., 1965.

3. G. Lusztig, Some examples of square integrable representations of semisimple p-adic groups, Trans. Amer. Math. Soc. 277 (1983), 623-653.

4. G. Segal, Equivariant K-theory, Inst. Hautes Études Sci. Publ. Math. 34 (1968), 129-151.

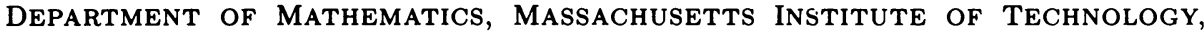
CAMBRIdGe, MASSACHUSETTS 02139 